\title{
Which parameters are important before attempting chronic total occlusions recanalization?
}

\author{
Leszek Bryniarski ${ }^{1}$, Krzysztof L. Bryniarski \\ 1 Department of Cardiology, Interventional Electrocardiology and Hypertension, Jagiellonian University Medical College, \\ University Hospital, Krakow, Poland \\ 2Department of Interventional Cardiology, Institute of Cardiology, Jagiellonian University Medical College, John Paul II Hospital, Krakow, \\ Poland
}

Adv Interv Cardiol 2016; 12, 2 (44): 96-98

DOI: 10.5114 /aic.2016.59358

Even in the era of advanced skills of operators of chronic total occlusions (CTO) combined with new sophisticated technologies and devices, percutaneous coronary interventions ( $\mathrm{PCl}$ ) of CTO remain challenging. These procedures are technically complex. Thus, the risk of failure and complications is very dependent on technical skills of the operator and vary from study to study. There are also some controversies, fortunately year by year less frequent, mainly due to the sharing of knowledge in the cardiology community, creating appropriate indications for CTO revascularization [1, 2]. Finally, there are still some uncertainties regarding benefits of the CTO procedure, but the growing number of studies reporting good procedural, functional and, most importantly, a positive effect on long-term outcome, shifts the balance toward benefits. Despite the lack of randomized trials, in a recent meta-analysis of 25 studies published from 1990 to 2014 with 28,486 patients (29,315 CTO PCI procedures) with a mean follow-up of 3.11 years (range 6 months to 12 years) Christakopoulos from the E. Brilakis group elegantly showed that successful CTO PCI compared with failed procedures are associated with a lower risk of death, stroke and coronary artery bypass grafting and less recurrent angina pectoris [3].

When dealing with CTO we should take into account two very important aspects - the technical aspect and the impact of prognosis of the patients. If the qualification for the procedure is proper, according to the angiographic point of view, the chance of success is greater. Moreover, if the patient has a proper clinical indication (i.e. a sufficient amount of ischemia or viability) both aspects may lead to a better outcome during the procedure and follow-up.
In the previous issue of Advances in Interventional Cardiology, Baykan et al. tried to find factors which adversely influence the success rate of CTO PCI [4]. After analysis of 173 procedures they concluded that bridge collaterals, severe calcification and tortuosity, tandem/ multiple occlusions were independent predictors of unsuccessful CTO revascularization, while the presence of micro-channels was a predictor of success. The Turkish authors reported a $83.2 \%$ success rate, acceptable but slightly lower than that presented by highly specialized CTO centers. Some of the predictors of success are similar to variables used in the J-CTO score (calcification, tortuosity, tandem and multiple occlusions may correspond to long occlusions), some not, but this could be due to statistical analysis in relatively small groups of patients. Surprisingly, the J-CTO score was not an independent predictor of success, although it was significantly higher (2.3 vs. $1.9 ; p=0.006)$ in the failure group. Very disturbing is the high rate of reported complications (23 patients, $13.3 \%)$, but it may depend on the definition of coronary dissections in 16 patients as complications without specifying which kinds of dissection were included (donor vessel in retrograde?, proximal part of the vessel in antegrade?). Complications specific for $\mathrm{CTO} \mathrm{PCl}$, i.e. coronary perforations occurred in $6(3.5 \%)$ cases and 1 (0.6\%) cardiac tamponade, are consistent with other reports $[5,6]$.

The most popular index used in classification of the severity of CTO is the J-CTO score. This index was introduced by Morino in 2011 and originally was developed to predict the likelihood of successful guidewire crossing within $30 \mathrm{~min}$ [7]. Independent angiographic predictors of failure (each given 1 point) that made up the J-CTO score included prior failed attempt, angiographic evi-

\section{Corresponding author:}

Krzysztof L. Bryniarski MD, Department of Interventional Cardiology, Institute of Cardiology, Jagiellonian University Medical College, John Paul II Hospital, 80 Prądnicka St, 31-202 Krakow, Poland, phone: +48 603516 797, e-mail: kbrynia@gmail.com

Received: 3.03.2016, accepted: 3.03.2016. 
dence of heavy calcification, bending within the occluded segment, blunt proximal stump, and occlusion length $>20 \mathrm{~mm}$. Chronic total occlusions were then graded as easy, intermediate, difficult and very difficult (J-CTO scores of $0,1,1$, and $\geq 3$ respectively) (Table I). Since then, the J-CTO score has been found to predict overall likelihood of CTO PCI success. High J-CTO scores have been shown to correlate with lesion complexity, and may account for the paradox of a stagnant CTO PCI success rate over time, which was due to intervening on increasingly complex CTO $[8,9]$. The J-CTO score is easy to calculate. It is also a widely used, validated, preprocedural clinical and angiographic scoring system to assess lesion complexity. Recently Tanaka et al. showed that, in addition to crossing times, the J-CTO score appears to correlate with long-term success [10].

Other investigators try to use coronary computed tomography angiography (CCTA) to improve on the J-CTO score [11]. Opolski et al. reported on a CCTA-derived scoring system in a cohort of 240 CTO PCI lesions from 4 European centers. They assigned 1 point for each independent predictor of successful guidewire crossing within $30 \mathrm{~min}$. The points are then assumed to yield the CT-RECTOR score. Chronic total occlusions lesions were categorized as easy (score 0), intermediate (score 1), difficult (score 2 ), and very difficult (score $>3$ ). In this study, independent predictors of failure derived from CCTA analysis included occlusion length $>20 \mathrm{~mm}$, multiple occlusions, blunt stump, bending, and severe calcification in the CTO segment. Clinical predictors of failure included a previously failed attempt at percutaneous revascularization and duration of CTO > 12 months or unknown duration of occlusion. Using this score, the probability of successful guidewire crossing within 30 min for each group (from easy to very difficult) was $95 \%, 88 \%, 57 \%$ and $22 \%$ respectively. We should remember that the use of CCTA is very limited although it can reliably visualize CTO length, morphology and composition; moreover, there is no evidence to suggest that it helps in increasing the success rate. Accordingly to the EuroCTO Club consensus, CCTA can be recommended for complex CTO lesions with an expected success rate $<50 \%$ and in cases of repeat pro- cedures after initial СTO recanalization failure [5]. The decision to revascularize a CTO is a clinical one, on the basis of symptoms, myocardial viability, and patient preference, and should not be based on the ease or difficulty of the case. As such, the CT-RECTOR score is useful in identifying highly complex cases that should be avoided by operators early in their CTO PCI learning curve and referred to expert centers [12].

In our opinion in 2016 still the J-CTO score is most useful in grading the severity of СTO, and its simplicity provides particular utility in daily clinical practice. Of course, analysis of one's own material is important, as presented by Baykan et al., and also has a role in the learning process, helping in understanding which factors can influence the success rate.

In recent years a new benchmark for CTO success rates over $90 \%$ has been established. Anatomy dictates how and who should perform the CTO PCI, not whether the CTO PCI should be attempted. Similarly, lesion complexity no longer dictates the feasibility of CTO $\mathrm{PCl}$, but the strategy for successful CTO revascularization. According to the opinion of Walsh and Hanratty, the key elements for successful CTO PCI are the following: 1) the development of a program with full team involvement and moving away from ad hoc $\mathrm{CTO} \mathrm{PCl}$; 2) physician and staff education to help understand lesion complexity, techniques and objectives that have procedural safety as the central core; 3 ) learning from live or recorded cases and workshops to enhance understanding of equipment and techniques; and 4) proctoring - preferably with the proctor able to scrub-in for the case to move beyond technically challenging or potentially limiting steps in the procedure and thus allow the host physician to gain hands-on experience in all aspects of the CTO PCI, particularly the parts of the procedure that may not have been reached otherwise [13].

One of the authors of this commentary, as a member of EuroCTO Club, has also been involved in teaching and training for many years. In our opinion the goal should be to increase the total number of patients offered a CTO PCI rather than focusing on marginal increases in success rates in expert and specialized centers. Thereby,

Table I. J-CTO Score sheet

\begin{tabular}{lccl} 
Parameter & $\mathbf{0}$ points & $\mathbf{1}$ point & \multicolumn{1}{c}{ Explanation } \\
\hline Entry shape & Tapered & Blunt & $\begin{array}{l}\text { Entry with any tapered tip or dimple indicating direction of true lumen is categorized } \\
\text { as tapered }\end{array}$ \\
\hline Calcification & Absent & Present & $\begin{array}{l}\text { Regardless of severity, 1 point is assigned if any evident calcification is detected } \\
\text { within CTO segment }\end{array}$ \\
\hline Bending & $\leq 45^{\circ}$ & $>45^{\circ}$ & Any tortuosity separated from the CTO segment is excluded from this assessment \\
\hline Occlusion length & $<20 \mathrm{~mm}$ & $\geq 20 \mathrm{~mm}$ & $\begin{array}{l}\text { Using good collateral images, try to measure "true" distance of occlusion, which } \\
\text { tends to be shorter than the first impression }\end{array}$ \\
\hline Re-try lesion & No & Yes & $\begin{array}{l}\text { Re-try lesion is lesion previously attempted to recanalize but failed } \\
\text { No }\end{array}$
\end{tabular}

J-CTO score sheet by Morino et al. [7]. Points indicate difficulty of recanalization: 0 points - easy, 1 point - intermediate, 2 points - difficult, 3 or more points - very difficult. 
a greater total number of patients will be treated, leading to a reduction in symptoms and an improved quality of life and, most important, improving the prognosis for a much broader cohort of CTO patients. This year we can see some light at the end of the tunnel, confirming the efficacy of our efforts. According to unpublished data of the Association of Cardiovascular Interventions of the Polish Cardiac Society (ACVI), during the first 6 months of 2014 in Poland 601 CTO procedures were performed, with a $54 \%$ success rate. This efficacy has remained stable during the past years. But the data from 2015 are very satisfactory, showing an increase of the overall success rate in CTO procedures in Poland up to $68 \%$. We believe that in the future this trend will be preserved and our efforts in education of interventional cardiologists will lead to better prognosis of our patients.

\section{Conflict of interest}

The authors declare no conflict of interest.

\section{References}

1. Bryniarski KL, Zabojszcz M, Dębski G, et al. What do Polish interventional cardiologists know about indications and qualification for recanalization of chronic total coronary artery occlusions? Kardiol Pol 2015; 73: 722-9.

2. Bryniarski KL, Zabojszcz M, Dębski G, et al. Knowledge of chronic total occlusion among Polish interventional cardiologists. Postep Kardiol Inter 2015; 11: 89-94.

3. Christakopoulos GE, Christopoulos G, Carlino M, et al. Metaanalysis of clinical outcomes of patients who underwent percutaneous coronary interventions for chronic total occlusions. Am J Cardiol 2015; 115: 1367-75.

4. Baykan AO, Gür M, Acele A, et al. Predictors of successful percutaneous coronary intervention in chronic total coronary occlusions. Adv Interv Cardiol 2016; 12: 17-24.

5. Sianos G, Werner GS, Galassi A, et al. Recanalisation of chronic total coronary occlusions: 2012 consensus document from the EuroCTO club. Eurolntervention 2012; 8: 139-45.

6. Bryniarski L, Surowiec S, Klima $Ł$, et al. Recanalization of coronary chronic total occlusions by retrograde approach: the first experience in Poland. Kardiol Pol 2015; 73: 167-76.

7. Morino Y, Abe M, Morimoto T, et al. Predicting successful guidewire crossing through chronic total occlusion of native coronary lesions within 30 minutes: the J-CTO (Multicenter CTO Registry in Japan) score as a difficulty grading and time assessment tool. JACC Cariovasc Interv 2011; 4: 213-21.

8. Nombela-Franco L, Urena $M$, Jerez-Valero $M$, et al. Validation of the J-chronic total occlusion score for chronic total occlusion percutaneous coronary intervention in an independent contemporary cohort. Circ Cardiovasc Interv 2013; 6: 635-43.

9. Syrseloudis D, Secco GG, Barrelo EA, et al. Increase in J-CTO lesion complexity explains the disparity between recanalization success and evolution of chronic total occlusion strategies: insights from a single-centre 10-year experience. Heart 2013; 99: 474-9.

10. Tanaka H, Morino Y, Mitsuru A, et al. Impact of J-CTO score on procedural outcome and target lesion revascularisation after percutaneous coronary intervention for chronic total occlusion: a substudy of the J-СTO Registry (Multicentre CTO Registry in Japan. Eurolntervention 2016; 11: 981-8.

11. Opolski MP, Achenbach S, Schuhbäck A, et al. Coronary computed tomographic prediction rule for time-efficient guidewire crossing through chronic total occlusion: insights from the CT-RECTOR multicentre registry (Computed Tomography Registry of Chronic Total Occlusion Revascularization). JACC Cardiovasc Interv 2015; 8: 257-67.

12. Karmpaliotis D, Green P. Chronic total occlusion percutaneous coronary intervention in 2014 beyond the J-CTO Score (Japanese Multicenter CTO Registry). Chance favors the prepared mind. JACC Cardiovasc Interv 2015; 2: 268-70.

13. Walsh SJ, Hanratty CG. Percutaneous coronary intervention for chronic total occlusions: time to move from the annex to mainstream? Eurolntervention 2016; 11: 974-6. 